\title{
Assessment Nurses Performance for Patients with Cancer Receiving Chemotherapy at South Egypt Cancer Institute at Assiut University
}

\author{
Sabra , H., E; El. Shemy, H., M ; Mohamed , M., A ; Mohamed, S., Sh \&Yousef H, R. \\ Assistance Lecturer- Nursing Administration Quena University Superversity. \\ Professor Of Nursing Administration, Faculty Of Nursing Ain Shams University . \\ Professor Of Clinical Oncology Faculty Of Medicine, Assiut University. \\ Assistant Professor Of Nursing Administration, Faculty Of Nursing, Assiut University
}

\begin{abstract}
:
The descriptive study aimed at assessment nurses performance for patients with cancer receiving chemotherapy at South Egypt Cancer Institute at Assiut University: Subjects included in the study were 65 nurses from male, female and private oncology units. The collection of data was achieved by using observation checklist: this tool aims at assess performance of the nurses who providing care for patients with cancer receiving chemotherapy. It was developed by the researcher based on review of related literature. This tool consisted of two parts. Part I: Entailed. Socio-demographic characteristics of the studied nurses such as age, sex, qualification, and years of experience. Part II: Entailed the nursing care for patients with cancer receiving chemotherapy. It includes 147 criteria under 10 heading that identify nursing care for patient with cancer receiving chemotherapy. The results of the study showed that nursing care provided for patients with cancer receiving chemotherapy were inadequate. Based on the finding of the study. It is recommended that the oncology unit should be staffed with qualified and adequate nurses who are to make assessment and to provide adequate nursing intervention during three day shifts according to patients needs.
\end{abstract}

\section{Key words: Patient with Cancer, Chemotherapy}

\section{Introduction:}

Cancer is not just one disease, but a large group of almost one hundred diseases. It is two main characteristics are uncontrolled growth of cell in the human body and the ability of these cells to migrate from the original site spread to distant sites. If the spread is not controlled, cancer result death (Wikipedia, 2009).

Chemotherapy is the treatment of cancer with drugs (anti-cancer drugs) that destroy cancer cells. It interferes with cell division in various possible ways, e. g. duplication of DNA or the separation of newly formed chromosomes. Most forms of chemotherapy target all rapidly dividing cells and are not specific for cancer (Vogel \& Costantino, 2006). Cancer chemotherapy has principally been used in the management of advanced or metastatic disease, following failed local therapies, or in disease for which no alternative therapy is effective (Day \& Adjei, 2006).

The patient's ability to cope with chemotherapy will be influenced by knowledge of the treatment and realistic expectations of side effects as the patient's ability to control or manage the side effects. Therefore the nurse plays an important role as supportive and educative, in order to allow individuals to cope with therapy and maintaining the highest possible quality of life (Hargrove \& Derstine 2006).

Nurses play a vital role regarding patients with cancer. This role includes, monitoring acute reaction and follow up care of these patients, and providing education, counseling and support as the patients facing change in their life. Also nurse play important role to help the patient to cope and adapt to their illness. Nurses develop intervention programs that take the form of psycho-educational preparation aimed at helping the patient to develop acceptance of self despite, the disability, to enhance their selfesteem and sense of control. Psycho-educational preparation is the primary intervention that is expected to raise the patients line of defense and reduce their anxiety. (Sabra, 2003).

Aim of the study: The study aimed at assessment nurses performance for patients with cancer receiving chemotherapy at South Egypt Cancer Institute at Assiut University

Subject and methods:

Setting: The study was conducted in male, female and private oncology units at south Egypt Cancer Institute at Assiut University.

Subjects. The total number of nurses included in the study were (65)

nurses, representing total coverage for all responded nurses in the study setting who are currently assigned and provide care for patients with cancer.

Data collection tools: The collection of data was achieved by using an observation checklist. This tool aims to assess performance of the nurses who providing care for patients with cancer receiving chemotherapy. It was developed by the researcher based on review of related literature (Cancer Care 
Ontario, 1996; Mohammed, 1999; American Cancer College of Surgeon, 2004; Joint Commission on Accreditation of Health Care Organization, 2005 and Association Commission Cancer Center Guidelines, 2006). This tool consisted of two parts.

Part I: This entailed Socio-demographic characteristics of the studied nurses such as age, sex, qualification, and years of experience.

Part II: It was developed to assess the performance of nurses who providing care for patients with cancer receiving chemotherapy through an initial list of performance. It includes 147 criteria under 10 heading that identify nursing care for patient with cancer receiving chemotherapy as the following. Assessment (15) items, laboratory investigation (6) items, handling chemotherapy (8) items, nursing care during receiving chemotherapy (58) items, supportive and psychological care (6) items, nutrition (11) items, skin care (6) items, neurotoxisty (10) items, extravasation (15) items and discharge (12) items.

Scoring system: Scoring system for checklist items was scored one when activity was done and scored zero when the activity was not done. The total score of the checklist was 147 .

\section{Assessing validity of observation checklist.}

It was ascertain by Jury who consisted of three categories.

1-Eight from Faculty of Nursing representing (four professors, two assistant professors and two lecturers) in the field of education from medical surgical and administration departments at Assiut and Ain Shams universities.

2- Medical faculty representing physicians (three professors, one assistant professor) working at the oncology department in Assiut University Hospital and (four lecturers) working at South Egypt Cancer Institute.

3-Thirteen head nurses representing nursing administrators in the field of services at South Egypt Cancer Institute and in different oncology units rendering nursing care for patients with cancer

4-Their opinions were elicited regarding the format layout of check list. However the Jury added some items which were done before data collection.

Operational design: This included the pilot study and the field work.

Pilot study: A pilot study was conducted on a sample of $10 \%$ of nurses aiming at determining the applicability of the tools, and to test the clarity of the language. It also helped to test the feasibility and suitability of the study settings, and to determine time needed to collect data needed for the study. Data obtained from the pilot were analyzed and necessary modifications were done.

Field work: Field work is conducting the study. Include the development of the observation checklist to collect data regarding nurses' performance (observation checklist).

Administration design:

To carry out the study at the selected settings an official permission was obtained from the Dean of South Egypt Cancer Institute, head of male, female, and private oncology units as well as from the director of nursing through a formal letter from the faculty of nursing at Assiut University. This was carried out at the time of observation process. Confidentiality of the obtained information was ensured after explanation of its purpose.

Statistical design:

Data were collected and fed into computer for analysis and presentation. Data were entered and analyzed by SPSS statistical analysis computer software. Data were presented descriptive statistics in the form of frequencies and percentages, as the reliability, data were paired $\boldsymbol{e} . g$. intra-rater. Statistical significance was considered at $\mathrm{p}$. value $<0.05$.

Reliability of the observed performance: Reliability of the observed nurses' performance was done by using:

1-The intra-rater reliability test which required the nurses to observe two times at the same time, but independently. This was applied to the nurses available at the time of the data collection.

2-The second test of reliability of the nurses' performance for the items included in the checklist was intra-rater reliability test-re-test technique, where the researcher repeated the process of observation to test stability of tool over the time of 14 days period between the first and the second time of observation. 


\section{Results:}

Table 1. Socio-demographic characteristics of nurses in the observation samples $(n=65)$.

\begin{tabular}{|l|c|c|}
\hline & Frequency & Percent \\
\hline Age (years): & \multicolumn{2}{|l|}{} \\
\hline$<25$ & 50 & 76.9 \\
\hline $25+$ & 15 & 23.1 \\
\hline Range & $19-32$ & \\
\hline Mean \pm SD & $23.4 \pm 2.3$ & 35.4 \\
\hline Sex: & \multicolumn{2}{|l|}{} \\
\hline Male & 23 & 64.6 \\
\hline Female & 42 & 100.0 \\
\hline Qualification: nursing secondary school diploma & 65 & 40.0 \\
\hline Unit: & \multicolumn{2}{|l|}{} \\
\hline Male & 26 & 36.9 \\
\hline Female & 24 & 23.1 \\
\hline Private & 15 & 49.2 \\
\hline Experience (years): & 32 & 50.8 \\
\hline$<5$ & 33 & \\
\hline $5+$ & $2.0-13.0$ & \\
\hline Range & $5.1 \pm 2.2$ \\
\hline Mean \pm SD & \multicolumn{2}{|l}{} \\
\hline
\end{tabular}

Table 2. Intra-rater test-re-test reliability of observation checklist for oncology nurses performance related to assessment and investigations $(n=65)$.

\begin{tabular}{|c|c|c|c|c|c|c|}
\hline \multirow{3}{*}{ Iten } & \multicolumn{4}{|c|}{ Time } & \multirow{3}{*}{$\begin{array}{c}\mathbf{X}^{2} \\
\text { Test }\end{array}$} & \multirow{3}{*}{$\begin{array}{c}p \text { - } \\
\text { value }\end{array}$} \\
\hline & \multicolumn{2}{|c|}{$\mathbf{1}$} & \multicolumn{2}{|c|}{2} & & \\
\hline & No. & $\%$ & No. & $\%$ & & \\
\hline \multicolumn{7}{|c|}{$\begin{array}{l}\text { 1. The assessment activities done by the oncology nurse } \\
\text { are: }\end{array}$} \\
\hline \multicolumn{7}{|c|}{$\begin{array}{l}\text { 1.1. Collect socio-demographic data as age, occupation, } \\
\text { income }\end{array}$} \\
\hline \multicolumn{7}{|c|}{ 1.1.1. Assessment general condition: } \\
\hline 1.1.1.1. Height & 31 & 47.7 & 44 & 67.7 & 5.33 & $0.02 *$ \\
\hline 1.1.1.2. Weight & 4 & 6.2 & 9 & 13.8 & 2.14 & 0.4 \\
\hline 1.1.1.3. Surface area & 32 & 49.2 & 40 & 61.5 & 1.99 & 0.16 \\
\hline \multicolumn{7}{|l|}{ 1.1.2. Assessment vital signs: } \\
\hline 1.1.2.1. Pulse & 0 & 0.0 & 0 & 0.0 & 0.00 & 1.00 \\
\hline 1.1.2.2. Respiration & 16 & 42.6 & 24 & 36.9 & 2.32 & 0.13 \\
\hline 1.1.2.3. Temperature & 56 & 86.2 & 63 & 96.9 & 4.87 & $0.03 *$ \\
\hline 1.1.2.4. BP & 39 & 60.0 & 45 & 69.2 & 1.21 & 0.27 \\
\hline \multicolumn{7}{|c|}{$\begin{array}{l}\text { 1-2 Assessment patient history for the present problem } \\
\text { from: }\end{array}$} \\
\hline 1.2.1. Patient & 53 & 81.5 & 59 & 90.6 & 2.32 & 0.13 \\
\hline 1.2.2. Significant others & 37 & 56.9 & 39 & 60.0 & 0.13 & 0.053 \\
\hline \multicolumn{7}{|c|}{ 1.3. Assessment the medical history such as: } \\
\hline 1.3.1. Patients complaints & 21 & 32.3 & 27 & 41.5 & 1.19 & 0.28 \\
\hline 1.3.2. Diabetes & 25 & 38.5 & 36 & 55.4 & 3.74 & 0.53 \\
\hline 1.3.3. Hypertension & 43 & 66.2 & $\overline{53}$ & 81.5 & 3.98 & 0.046 \\
\hline 1.3.4. Heart disease & 7 & 10.8 & 23 & 35.4 & 11.09 & $0.001 *$ \\
\hline
\end{tabular}




\begin{tabular}{|c|c|c|c|c|c|c|}
\hline \multirow{3}{*}{ Items } & \multicolumn{4}{|c|}{ Time } & \multirow{3}{*}{$\begin{array}{c}\mathbf{X}^{2} \\
\text { Test }\end{array}$} & \multirow{3}{*}{$\begin{array}{c}p- \\
\text { value }\end{array}$} \\
\hline & \multicolumn{2}{|c|}{1} & \multicolumn{2}{|c|}{2} & & \\
\hline & No. & $\%$ & No. & $\%$ & & \\
\hline 1.3.5. Psychological condition & 2 & 3.1 & 2 & 3.1 & Fisher & 1.00 \\
\hline 1.3.6. Other chronic diseases & 6 & 9.2 & 9 & 13.8 & 1.19 & 0.28 \\
\hline \multicolumn{7}{|c|}{$\begin{array}{l}\text { 2. Nursing care in relation to performing investigations } \\
\text { includes: }\end{array}$} \\
\hline 2.1. Date ordered is written on the request & 58 & 89.2 & 62 & 95.4 & 1.73 & 0.19 \\
\hline 2.2. Obtain specimen accurately & 30 & 46.2 & 46 & 70.8 & 8.11 & $0.004 *$ \\
\hline 2.3. Record time of obtaining specimen & 5 & 7.7 & 13 & 20.0 & 4.13 & $0.04 *$ \\
\hline 2.4. Record test type & 63 & 96.9 & 65 & 100.0 & Fisher & 0.50 \\
\hline 2.5. Follow sent specimen & 30 & 46.2 & 46 & 70.8 & 8.11 & 0.19 \\
\hline 2.6. Follow results of specimen & 7 & 10.8 & 10 & 15.4 & 0.61 & 0.44 \\
\hline
\end{tabular}

(*) Statistically significant at $p<0.05$

Table 3. Intra-rater test-re-test reliability of observation checklist for oncology nurses performance related to handling chemotherapeutic agents $(n=65)$

\begin{tabular}{|c|c|c|c|c|c|c|}
\hline \multirow{3}{*}{ Items } & \multicolumn{4}{|c|}{ Time } & \multirow{3}{*}{$\begin{array}{c}\mathbf{X}^{2} \\
\text { Test }\end{array}$} & \multirow{3}{*}{$p$-value } \\
\hline & \multicolumn{2}{|c|}{1} & \multicolumn{2}{|c|}{2} & & \\
\hline & No. & $\%$ & No. & $\%$ & & \\
\hline \multicolumn{7}{|l|}{ 3. Nursing care before handling chemotherapeutic agents: } \\
\hline 3.1. Wear disposable latex gloves & 37 & 56.9 & 39 & 60.0 & 0.13 & 0.72 \\
\hline 3.2. Mask to cover nose and mouth & 0 & 0.0 & 0 & 0.0 & 0.0 & 0.0 \\
\hline 3.3. Wear eye protectors & 0 & 0.0 & 1 & 1.5 & Fisher & 1.00 \\
\hline 2.4. Avoid spills of drugs & 55 & 84.6 & 57 & 87.7 & 0.26 & 0.61 \\
\hline 3.5. Aerosolization when drawing up from a vial & 52 & 80.0 & 58 & 89.2 & 2.13 & 0.14 \\
\hline 3.6. Expel excess drugs from syringes into air & 55 & 84.6 & 59 & 90.8 & 1.14 & 0.29 \\
\hline 3.7. Dispose of needles and syringes in special containers & 44 & 67.7 & 58 & 89.2 & 8.92 & $0.003^{*}$ \\
\hline 3.8. Always be familiar with the drugs & 58 & 89.2 & 63 & 96.9 & Fisher & 0.16 \\
\hline
\end{tabular}

(*) Statistically significant at $p<0.05$

Table 4. Intra-rater test-re-test reliability of observation checklist for oncology nurses performance related to assessment of infection, fever, anemia, and thrombocytopenia $(n=65)$.

\begin{tabular}{|c|c|c|c|c|c|c|}
\hline \multirow{3}{*}{ Items } & \multicolumn{4}{|c|}{ Time } & \multirow{3}{*}{$\begin{array}{c}X^{2} \\
\text { Test }\end{array}$} & \multirow{3}{*}{$\begin{array}{c}p- \\
\text { value }\end{array}$} \\
\hline & \multicolumn{2}{|c|}{1} & \multicolumn{2}{|c|}{2} & & \\
\hline & No. & $\%$ & No. & $\%$ & & \\
\hline \multicolumn{7}{|l|}{$\begin{array}{l}\text { 4. The nursing care done when patient is receiving } \\
\text { chemotherapy includes: }\end{array}$} \\
\hline \multicolumn{7}{|l|}{ 4.1. Identification of infection: } \\
\hline \multicolumn{7}{|l|}{ 4.1.1. Inspect skin and mucus membranes daily especially } \\
\hline Mouth & 50 & 76.9 & 57 & 87.7 & 2.59 & 0.11 \\
\hline Axilla & 9 & 13.8 & 12 & 18.5 & 0.51 & 0.47 \\
\hline Perineum & 0 & 0.0 & 3 & 4.6 & Fisher & 0.24 \\
\hline 4.1.2. Auscultate respiratory rate & - & - & - & - & - & - \\
\hline \multicolumn{7}{|l|}{ 4.1.3. Monitor regularly: } \\
\hline Temperature & 25 & 38.5 & 36 & 55.4 & 3.74 & 0.53 \\
\hline Pulse & 0 & 0.0 & 2 & 3.1 & Fisher & 0.50 \\
\hline Respiration & 0 & 0.0 & 3 & 4.6 & Fisher & 0.24 \\
\hline \multicolumn{7}{|l|}{ 4.1.4. Monitor neutrophil count: } \\
\hline A count of 500 to $1000 / \mathrm{mm}$ indicates moderate risk of infection & 23 & 35.4 & 26 & 40.0 & 0.29 & 0.59 \\
\hline
\end{tabular}




\begin{tabular}{|c|c|c|c|c|c|c|}
\hline \multirow{3}{*}{ Items } & \multicolumn{4}{|c|}{ Time } & \multirow{3}{*}{$\begin{array}{c}\mathbf{X}^{2} \\
\text { Test }\end{array}$} & \multirow{3}{*}{$\begin{array}{c}p- \\
\text { value }\end{array}$} \\
\hline & \multicolumn{2}{|c|}{$\mathbf{1}$} & \multicolumn{2}{|c|}{2} & & \\
\hline & No. & $\%$ & No. & $\%$ & & \\
\hline A count of less than $500 / \mathrm{mm}$ indicates sever risk of infection & 24 & 36.9 & 31 & 47.7 & 1.54 & 0.21 \\
\hline \multicolumn{7}{|l|}{ 4.2. Assessment of fever: } \\
\hline \multicolumn{7}{|l|}{ Assessment of fever and signs of infection } \\
\hline If fever is $\leq 382$ comfort measure are provided & 43 & 66.2 & 53 & 81.5 & 3.98 & 0.046 \\
\hline If is $\geq 382$ the physician should be notified & 59 & 90.8 & 62 & 95.4 & Fisher & 0.49 \\
\hline \multicolumn{7}{|l|}{ 4.3. Assessment for anemia: check: } \\
\hline 4.3.1. Hemoglobin level & 59 & 90.8 & 62 & 95.4 & 0.10 & 0.75 \\
\hline 4.3.2. Platelets count & 12 & 18.5 & 12 & 18.5 & 0.00 & 1.00 \\
\hline 4.3.3. Differential count & 6 & 9.2 & 9 & 13.8 & 0.68 & 0.41 \\
\hline \multicolumn{7}{|l|}{ 4.4. Assessment for thrombocytopenia } \\
\hline \multicolumn{7}{|l|}{ 4.4.1. Assess symptoms and signs of bleeding such as: } \\
\hline Petechia & 24 & 36.9 & 30 & 46.2 & 1.14 & 0.29 \\
\hline Ecchymosis & 16 & 24.6 & 17 & 26.2 & 0.029 & 0.44 \\
\hline Consciousness level & 49 & $\overline{75.4}$ & 53 & 81.5 & 0.73 & 0.39 \\
\hline Headache & 51 & 78.5 & 56 & 86.2 & 1.32 & 0.25 \\
\hline Active bleeding & 58 & 89.2 & 64 & 98.5 & Fisher & 0.06 \\
\hline
\end{tabular}

(*) Statistically significant at $p<0.05$

Table 5. Intra-rater test-re-test reliability of observation checklist for oncology nurses performance related to gastro-intestinal problems $(n=65)$

\begin{tabular}{|c|c|c|c|c|c|c|}
\hline \multirow{3}{*}{ Items } & \multicolumn{4}{|c|}{ Time } & \multirow{3}{*}{$\begin{array}{c}\mathbf{X}^{2} \\
\text { Test }\end{array}$} & \multirow{3}{*}{$\begin{array}{c}p- \\
\text { value }\end{array}$} \\
\hline & \multicolumn{2}{|c|}{$\mathbf{1}$} & \multicolumn{2}{|c|}{2} & & \\
\hline & No. & $\%$ & No. & $\%$ & & \\
\hline \multicolumn{7}{|l|}{$\begin{array}{l}\text { 4.5. Nursing care regarding gastrointestinal tract } \\
\text { includes: }\end{array}$} \\
\hline \multicolumn{7}{|l|}{4.51 Nursing care for nausea /vomiting: } \\
\hline 4.5.1.1. Observe number of emesis/24hours & 51 & 78.5 & 56 & 86.2 & 1.32 & 0.25 \\
\hline 4.5.1.2. Observe oral intake /24hours & 17 & 26.2 & 21 & 32.3 & 0.29 & 0.44 \\
\hline 4.5.1.3. Give antiemitics treatment as ordered & 29 & 90.8 & 58 & 89.2 & 0.09 & 0.77 \\
\hline 4.5.1.4. Observe weight loss & 20 & 30.8 & 22 & 33.8 & 0.14 & 0.71 \\
\hline \multicolumn{7}{|l|}{ 4.5.2. Nursing care for diarrhea: } \\
\hline $\begin{array}{l}\text { 4.5.2.1. Observe number of defecation } / 24 \text {, character, } \\
\text { consistency }\end{array}$ & 54 & 83.1 & 54 & 83.1 & 0.00 & 1.00 \\
\hline 4.5.2.2. Identify food allergies & 18 & 27.7 & 22 & 33.8 & 0.58 & 0.45 \\
\hline 4.5.2.3. Fever/24 hrs & 55 & 84.6 & 62 & 95.4 & 4.19 & $0.04 *$ \\
\hline 4.5.2.4. Give anti diarrheal medication & 55 & 84.6 & 58 & 89.2 & 0.61 & 0.44 \\
\hline 4.5.2.5. Assess patient weight loss & 21 & 32.3 & 27 & 41.5 & 1.19 & 0.28 \\
\hline 4.5.2.6. Determine level of activity & 7 & 10.8 & 9 & 13.8 & 0.29 & 0.59 \\
\hline 4.5.2.7. Assess vital signs to rule out dehydration & 32 & 49.2 & 41 & 63.1 & 2.53 & 0.11 \\
\hline $\begin{array}{l}\text { 4.5.2.8. Provide comfort measures such as hygiene to } \\
\text { perineum after bowel movement }\end{array}$ & 2 & 3.1 & 2 & 3.1 & Fisher & 1.00 \\
\hline 4.5.2.9. Perform stool culture as ordered & 28 & 43.1 & 33 & 50.8 & 0.77 & 0.38 \\
\hline \multicolumn{7}{|l|}{ 4.5.3. Nursing care for constipation: } \\
\hline 4.5.3.1.Observe for lack of appetite, or energy & 12 & 1805 & 16 & 24.6 & 0.73 & 0.39 \\
\hline $\begin{array}{l}\text { 4.5.3.2. Encourage small, frequent meals, high fiber diet, } \\
\text { increased fluid intake }\end{array}$ & 16 & 24.6 & 17 & 26.2 & 0.04 & 0.84 \\
\hline 4.5.3.3.Weekly weights & 47 & 72.3 & 53 & 81.5 & 1.56 & 0.21 \\
\hline 4.5.3.4. Appetite stimulation as order & 58 & 89.2 & 63 & 96.9 & Fisher & 0.16 \\
\hline
\end{tabular}




\begin{tabular}{|c|c|c|c|c|c|c|}
\hline \multirow{3}{*}{ Items } & \multicolumn{4}{|c|}{ Time } & \multirow{3}{*}{$\begin{array}{c}\mathbf{X}^{2} \\
\text { Test }\end{array}$} & \multirow{3}{*}{$\begin{array}{c}p- \\
\text { value }\end{array}$} \\
\hline & \multicolumn{2}{|c|}{1} & \multicolumn{2}{|c|}{2} & & \\
\hline & No. & $\%$ & No. & $\%$ & & \\
\hline $\begin{array}{l}\text { 4.5.3.5. Gives patient oral softeners daily according to } \\
\text { doctor order }\end{array}$ & 43 & 66.2 & 53 & 81.5 & 3.98 & 0.046 \\
\hline $\begin{array}{l}\text { 4.5.3.6. Administers suppositories minutes before defecation } \\
\text { time as order }\end{array}$ & 9 & 1308 & 13 & 20.0 & 0.88 & 0.35 \\
\hline \multicolumn{7}{|l|}{ 4.5.4. Nursing care for stomatitis: } \\
\hline 4.5.4.1. Observe dry mouth & 54 & 83.1 & 58 & 89.2 & 1.03 & 0.31 \\
\hline 4.5.4.2. Observe bleeding gum & 42 & 64.6 & 45 & 69.2 & 0.31 & 0.58 \\
\hline $\begin{array}{l}\text { 4.5.4.3. Provide mouth care every } 4 \text { to } 6 \text { hours with normal } \\
\text { saline }\end{array}$ & 3 & 4.6 & 12 & 18.5 & 6.10 & $0.01 *$ \\
\hline 4.5.4.4. Avoid lemon or glycerin swabs & 2 & 3.1 & 9 & 13.8 & 4.87 & $0.03 *$ \\
\hline $\begin{array}{l}\text { 4.5.4. 5. Apply topical viscous anesthetic as lidocaine before } \\
\text { meals as ordered }\end{array}$ & 21 & 32.3 & 27 & 41.5 & 1.19 & 0.28 \\
\hline $\begin{array}{l}\text { 4.5.4.6. Consult dietitian to provide blend of food at } \\
\text { medium temperature }\end{array}$ & 3 & 4.6 & 11 & 16.9 & 5.12 & 0.02 \\
\hline
\end{tabular}

(*) Statistically significant at $p<0.05$

Table 6. Intra-rater test-re-test reliability of observation checklist for oncology nurses performance related to pulmonary and urinary systems $(n=65)$

\begin{tabular}{|c|c|c|c|c|c|c|}
\hline \multirow{3}{*}{ Items } & \multicolumn{4}{|c|}{ Time } & \multirow{3}{*}{$\begin{array}{c}\mathbf{X}^{2} \\
\text { Test }\end{array}$} & \multirow{3}{*}{$\begin{array}{c}p- \\
\text { value }\end{array}$} \\
\hline & \multicolumn{2}{|c|}{1} & \multicolumn{2}{|c|}{2} & & \\
\hline & No. & $\%$ & No. & $\%$ & & \\
\hline \multicolumn{7}{|l|}{ 4.6. Nursing care for pulmonary system includes: } \\
\hline 4.6.1. Auscultate lungs & 0 & 0.0 & 1 & 1.5 & Fisher & 1.00 \\
\hline \multicolumn{7}{|l|}{ 4.6.2. Observe for: } \\
\hline Shortness of breath & 25 & 38.5 & 34 & 52.3 & 2.51 & 0.11 \\
\hline Cough & 57 & 87.7 & 63 & 96.9 & 3.90 & 0.048 \\
\hline Presence of sputum & 25 & 38.5 & 36 & 55.4 & 3.74 & 0.053 \\
\hline \multicolumn{7}{|l|}{ 4.7. Nursing care for urinary system includes: } \\
\hline \multicolumn{7}{|l|}{ 4.7.1. Observe urine for: } \\
\hline Color & 21 & 32.3 & 27 & 41.5 & 1.19 & 0.28 \\
\hline Turbidity & 14 & 21.5 & 17 & 26.2 & 0.38 & 0.54 \\
\hline Amount & 64 & 98.5 & 65 & 100.0 & Fisher & 1.00 \\
\hline $\begin{array}{l}\text { 4.7.2. Evaluate renal function by either serum creatinine or } 24 \\
\text { hours urine for creatinine clearance, urea, and urine analysis }\end{array}$ & 6 & 9.2 & 11 & 16.9 & 1.69 & 0.19 \\
\hline 4.7.3. Record intake and output & 58 & 89.2 & 60 & 92.3 & 0.37 & 0.54 \\
\hline \multicolumn{7}{|l|}{$\begin{array}{l}\text { 9.7.4. Monitor the signs and symptoms of urinary tract } \\
\text { infection: }\end{array}$} \\
\hline Frequency & 25 & 38.5 & 26 & 40.0 & 0.03 & 0.86 \\
\hline Dysuria & 19 & 29.2 & 26 & 40.0 & 1.67 & 0.2 \\
\hline 4.7.5. Gives adequate fluid intake & 60 & 92.3 & 64 & 98.5 & Fisher & 0.21 \\
\hline 4.7.6. Puts schedule toliting/2hrs & 0 & 0.0 & 0 & 0.0 & 0.00 & 1.00 \\
\hline 4.7.7. Fluid and electrolytes balance & 32 & 49.2 & 40 & 61.5 & 1.99 & 0.16 \\
\hline
\end{tabular}

(*) Statistically significant at $p<0.05$ 
Table 7. Intra-rater test-re-test reliability of observation checklist for oncology nurses performance related to psychological problems and nutrition $(n=65)$

\begin{tabular}{|c|c|c|c|c|c|c|}
\hline \multirow{3}{*}{ Items } & \multicolumn{4}{|c|}{ Time } & \multirow{3}{*}{$\begin{array}{c}\mathbf{X}^{2} \\
\text { Test }\end{array}$} & \multirow{3}{*}{$\begin{array}{c}p- \\
\text { value }\end{array}$} \\
\hline & \multicolumn{2}{|c|}{1} & \multicolumn{2}{|c|}{2} & & \\
\hline & No. & $\%$ & No. & $\%$ & & \\
\hline \multicolumn{7}{|l|}{$\begin{array}{l}\text { 5. Supportive and psychological nursing care rendered by the } \\
\text { oncology nurse for patient with cancer includes: }\end{array}$} \\
\hline 5.1. Explain the expected outcome and side effects for chemotherapy & 18 & 27.7 & 22 & 33.8 & 0.58 & 0.45 \\
\hline 5.2. Encourage verbalization and identification of fears & 5 & 7.7 & 6 & 9.2 & 0.10 & 0.75 \\
\hline 5.3. Help patient to explore ways to cope with fear & 2 & 3.1 & 4 & 6.2 & Fisher & 0.68 \\
\hline 5.4. Assess spiritual needs & 1 & 1.5 & 1 & 1.5 & Fisher & 1.00 \\
\hline 5.5. Offer support by active listening & 19 & 29.2 & 26 & 40.0 & 1.67 & 0.20 \\
\hline $\begin{array}{l}\text { 5.6. Offer hope in some form, and being there for patient and } \\
\text { relatives }\end{array}$ & 28 & 43.1 & 33 & 50.8 & 0.77 & 0.38 \\
\hline \multicolumn{7}{|l|}{ 6. Nursing care for improving nutrition includes: } \\
\hline 6.1. Assess taste alteration & 10 & 15.4 & 19 & 27.2 & 3.60 & 0.006 \\
\hline 6.2. Evaluate patient appetite & 17 & 26.2 & 21 & 32.3 & 0.29 & 0.44 \\
\hline 6.3. Give instructions for improving appetite & 37 & 56.9 & 39 & 60.0 & 0.13 & 0.72 \\
\hline 6.4. Keep patient room free from bad odor & 11 & 16.9 & 25 & 38.5 & 7.53 & $0.006^{*}$ \\
\hline 6.5. Give mouth care before and after meals & 10 & 15.4 & 19 & 29.2 & 3.60 & 0.06 \\
\hline 6.6. Give ordered antiemetics before and after chemo/ radiotherapy & 52 & 80.0 & 59 & 90.8 & 3.02 & 0.08 \\
\hline 6.7. Give small frequent meals & 1 & 1.5 & 34 & 52.3 & 1.11 & 0.29 \\
\hline $\begin{array}{l}\text { 6.8. Encourage added calories in meals, vitamins, carbohydrates and } \\
\text { supplements between meals }\end{array}$ & 0 & 0.0 & 2 & 3.1 & Fisher & 0.50 \\
\hline 6.9. Advise patient to use plastic utensils instead of metals & 1 & 1.5 & 2 & 3.1 & Fisher & 1.00 \\
\hline 6.10. Advise patient to take protein from eggs, beans instead of meat & 1 & 1.2 & 6 & 9.2 & Fisher & 0.11 \\
\hline 6.11. Advise patient to increase sugar and sweets intake & 2 & 3.1 & 7 & 10.8 & Fisher & 0.16 \\
\hline
\end{tabular}

(*) Statistically significant at $p<0.05$

Table 8. Intra-rater test-re-test reliability of observation checklist for oncology nurses performance related to skin alteration and neurotoxicity $(n=65)$

\begin{tabular}{|c|c|c|c|c|c|c|}
\hline \multirow{3}{*}{ Items } & \multicolumn{4}{|c|}{ Time } & \multirow{3}{*}{$\begin{array}{c}X^{2} \\
\text { Test }\end{array}$} & \multirow{3}{*}{$\begin{array}{c}p- \\
\text { value }\end{array}$} \\
\hline & \multicolumn{2}{|c|}{1} & \multicolumn{2}{|c|}{2} & & \\
\hline & No. & $\%$ & No. & $\%$ & & \\
\hline \multicolumn{7}{|l|}{ 7. Nursing care of skin alteration include: } \\
\hline 7.1. Provide opportunities to talk about change in body image & 7 & 10.8 & 9 & 13.8 & 0.29 & 0.59 \\
\hline 7.2. Persuade patient that these changes are reversible & 16 & 24.6 & 28 & 43.1 & 4.95 & $0.03 *$ \\
\hline $\begin{array}{l}\text { 7.3. Hair loss can be minimized by use of scalp } \\
\text { sphygmomanometer }\end{array}$ & 1 & 1.5 & 4 & 6.2 & Fisher & 0.37 \\
\hline $\begin{array}{l}\text { 7.4. Apply scalp tourniquet around the hairline before, during, } \\
\text { and for } 20 \text { minutes after infusion of the drug }\end{array}$ & 0 & 0.0 & 1 & 1.5 & Fisher & 1.00 \\
\hline $\begin{array}{l}\text { 7.5. Advise using hat or turban to cover head during period of } \\
\text { hair loss }\end{array}$ & 4 & 6.2 & 13 & 20.0 & 5.48 & $0.02 *$ \\
\hline $\begin{array}{l}\text { 7.6. Inform patient that the change in nail color is expected } \\
\text { during chemotherapy }\end{array}$ & 5 & 7.7 & 15 & 23.1 & 5.91 & $0.02 *$ \\
\hline \multicolumn{7}{|l|}{ 8. Nursing care for neurotoxicity includes: } \\
\hline 8.1. Instruct patient to report tinnitus & 7 & 10.8 & 15 & 23.1 & 3.50 & 0.06 \\
\hline 8.2. Instruct patient to report decreased hearing & 10 & 15.4 & 18 & 27.7 & 2.91 & 0.09 \\
\hline \multicolumn{7}{|l|}{ 8.3. Assess: } \\
\hline Numbness & 28 & 43.1 & 33 & 50.8 & 0.77 & 0.38 \\
\hline Tingling in extremities & 16 & 24.6 & 24 & 36.9 & 2.31 & 0.13 \\
\hline Decreased sensation & 4 & 6.2 & 11 & 16.9 & 3.69 & 0.054 \\
\hline
\end{tabular}




\begin{tabular}{|c|c|c|c|c|c|c|}
\hline \multirow{3}{*}{ Items } & \multicolumn{4}{|c|}{ Time } & \multirow{3}{*}{$\begin{array}{c}\mathbf{X}^{2} \\
\text { Test }\end{array}$} & \multirow{3}{*}{$\begin{array}{c}p- \\
\text { value }\end{array}$} \\
\hline & \multicolumn{2}{|c|}{$\mathbf{1}$} & \multicolumn{2}{|c|}{2} & & \\
\hline & No. & $\%$ & No. & $\%$ & & \\
\hline Foot drop & 0 & 0.0 & 2 & 3.1 & Fisher & 0.50 \\
\hline \multicolumn{7}{|l|}{ 8.4. Monitor and report: } \\
\hline Hoarseness & 2 & 3.1 & 6 & 9.2 & Fisher & 0.27 \\
\hline Jaw pain & 8 & 12.3 & 10 & 15.4 & 0.26 & 0.61 \\
\hline Decreased or absent deep tendon reflexes & $\overline{3}$ & 4.6 & 8 & 12.3 & 2.48 & 0.12 \\
\hline 8.5. Monitor and report depression & 54 & 83.1 & 58 & 89.2 & 1.03 & 0.31 \\
\hline
\end{tabular}

(*) Statistically significant at $p<0.05$

Table 9. Intra-rater test-re-test reliability of observation checklist for oncology nurses performance related to extravasation. $(n=65)$.

\begin{tabular}{|c|c|c|c|c|c|c|}
\hline \multirow{3}{*}{ Items } & \multicolumn{4}{|c|}{ Time } & \multirow{3}{*}{$\begin{array}{c}\mathbf{X}^{2} \\
\text { Test }\end{array}$} & \multirow{3}{*}{$p$-value } \\
\hline & \multicolumn{2}{|c|}{1} & \multicolumn{2}{|c|}{$\mathbf{2}$} & & \\
\hline & No. & $\%$ & No. & $\%$ & & \\
\hline \multicolumn{7}{|l|}{ 9- Nursing care when extravasation occurs includes: } \\
\hline 9.1. Stop chemotherapy administration & 64 & 98.5 & 64 & 98.5 & Fisher & 1.00 \\
\hline 9.2. Notify the physician & 12 & 18.8 & 12 & 18.8 & 0.51 & 0.47 \\
\hline $\begin{array}{l}\text { 9.3. Try to aspirate residual chemo-therapeutic agent from the } \\
\text { site and the cannula }\end{array}$ & 43 & 66.2 & 55 & 84.6 & 5.97 & $0.01^{*}$ \\
\hline $\begin{array}{l}\text { 9.4. Instill antidote by existing cannula if indicated } \\
\text { (hydrocortisone, hyaluronidase and sodium bicarbonate as } \\
\text { ordered) }\end{array}$ & 18 & 27.7 & 21 & 32.3 & 0.33 & 0.57 \\
\hline 9.5. No undue pressure is applied & 15 & 23.1 & 24 & 36.9 & 2.97 & 0.08 \\
\hline $\begin{array}{l}\text { 9.6. If unable to aspirate vesicant drug from tubing remove } \\
\text { cannula }\end{array}$ & 60 & 92.3 & 63 & 96.9 & Fisher & 0.44 \\
\hline \multicolumn{7}{|l|}{ 9.7. Apply: } \\
\hline Ethylchloride locally to the site & 23 & 35.4 & 26 & 40.0 & 0.29 & 0.59 \\
\hline Ice packs for at least $30 \mathrm{~min} / 4$ times /daily & 24 & 36.9 & 30 & 46.2 & 1.14 & 0.29 \\
\hline 9.8. Apply a bandage without pressure & 21 & 32.3 & 27 & 41.5 & 1.19 & 0.28 \\
\hline 9.9. Elevate the arm for days & 37 & 56.9 & 39 & 60.0 & 0.13 & 0.72 \\
\hline \multicolumn{7}{|l|}{ 9.10. Observe regularly for: } \\
\hline Pain & $\overline{14}$ & 21.5 & 38 & 58.5 & 18.46 & $<0.001^{*}$ \\
\hline Erythema & 37 & 56.9 & 39 & 60.0 & 0.13 & 0.72 \\
\hline Infection & 6 & 9.2 & 8 & 12.3 & 0.32 & 0.57 \\
\hline $\begin{array}{l}\text { 9.11. Consider plastic surgery consultation if pain persists after } \\
72 \mathrm{hrs}\end{array}$ & 5 & 7.7 & 11 & 16.9 & 2.57 & 0.11 \\
\hline 9.12. Document the occurrence in patient chart & 1 & 1.5 & 1 & 1.5 & Fisher & 1.00 \\
\hline
\end{tabular}

(*) Statistically significant at $p<0.05$

Table 10. Intra-rater test-re-test reliability of observation checklist for oncology nurses performance related to patient discharge $(n=65)$

\begin{tabular}{|c|c|c|c|c|c|c|}
\hline \multirow{3}{*}{ Items } & \multicolumn{4}{|c|}{ Time } & \multirow{3}{*}{$\begin{array}{c}\mathbf{X}^{2} \\
\text { Test }\end{array}$} & \multirow{3}{*}{$\begin{array}{c}p- \\
\text { value }\end{array}$} \\
\hline & \multicolumn{2}{|c|}{1} & \multicolumn{2}{|c|}{2} & & \\
\hline & No. & $\%$ & No. & $\%$ & & \\
\hline \multicolumn{7}{|l|}{ 10.The nursing care for discharge from hospital includes: } \\
\hline 10.1. Date of discharge & 65 & 100.0 & 65 & 100.0 & 0.00 & 1.00 \\
\hline 10.2. Time of discharge & 13 & 20.0 & 17 & 26.2 & 0.69 & 0.41 \\
\hline \multicolumn{7}{|l|}{ 10.4. Discharge instructions: } \\
\hline 10.4.1. Instruct patient to protect skin from cold, heat, or sun & 33 & 50.8 & 36 & 55.4 & 0.28 & 0.60 \\
\hline
\end{tabular}




\begin{tabular}{|c|c|c|c|c|c|c|}
\hline \multirow{3}{*}{ Items } & \multicolumn{4}{|c|}{ Time } & \multirow{3}{*}{$\begin{array}{c}\mathbf{X}^{2} \\
\text { Test }\end{array}$} & \multirow{3}{*}{$\begin{array}{c}p- \\
\text { value }\end{array}$} \\
\hline & \multicolumn{2}{|c|}{$\mathbf{1}$} & \multicolumn{2}{|c|}{2} & & \\
\hline & No. & $\%$ & No. & $\%$ & & \\
\hline $\begin{array}{l}10.42 \text { Instruct patient and family on procedure and visitation } \\
\text { restrictions and time of next cycle of chemotherapy }\end{array}$ & 27 & 41.5 & 27 & 41.5 & 0.00 & 1.00 \\
\hline \multicolumn{7}{|l|}{ 10.4.3. Instruct patient and his/her family about: } \\
\hline Side effects of treatment & 11 & 16.9 & 23 & 35.4 & 5.74 & $0.02 *$ \\
\hline Follow-up schedule & 37 & 56.9 & 41 & 63.1 & 0.51 & 0.47 \\
\hline Warning signs & 26 & 40.0 & 32 & 49.2 & 1.12 & 0.29 \\
\hline \multicolumn{7}{|l|}{$\begin{array}{l}\text { 10.6. Counseling regarding patient sexual and reproductive } \\
\text { functions: }\end{array}$} \\
\hline $\begin{array}{l}\text { 10. 6.1. Instruct patient to use an effective means of birth } \\
\text { control during the time of treatment and two years after } \\
\text { treatment }\end{array}$ & 0 & 0.0 & 1 & 1.5 & Fisher & 1.00 \\
\hline $\begin{array}{l}\text { 10. 6.2. Instruct patient to change time of day for sexual } \\
\text { relation if fatigue is a problem }\end{array}$ & 0 & 0.0 & 0 & 0.0 & -- & -- \\
\hline $\begin{array}{l}\text { 10. 6.3. Instruct patient to use vaginal lubricant before sexual } \\
\text { intercourse }\end{array}$ & 0 & 0.0 & 0 & 0.0 & -- & -- \\
\hline $\begin{array}{l}\text { 10.6.4. Assure that partner of the patient has no infectious } \\
\text { disease }\end{array}$ & 0 & 0.0 & 1 & 1.5 & Fisher & 1.00 \\
\hline ]0.7. Document patient condition at discharge & 1 & 1.5 & 1 & 1.5 & Fisher & 1.00 \\
\hline
\end{tabular}

(*) Statistically significant at $p<0.05$

Table 1 shows the sociodemographic characteristics of staff nurses observed. Data in this table reveals that the study nurses were 65 , more than two thirds $(76.9 \%)$ had their age less than 25 years, more than a half of them $(64.6 \%)$ were females while $(35.4 \%)$ of the observed nurses were males. It was noticed that all of them had nursing secondary school diploma, working in male, female, and private oncology units, about $(50.8 \%)$ of them had 5 and even more years of experience in nursing with a mean of 5.1 2.2.

Table 2 explains the results of intra-rater test, retest reliability of observation checklist for oncology nurses performance related to items of assessment and investigation. The data in this table shows that there is agreement upon the results obtained in the first and second times of observation except for the following items: assessing height, (P-value 0.02), assessing temperature (P-value 0.03 ), heart disease, $\mathrm{P}$ value (0.001), obtaining specimen accurately and recording time of obtaining specimen, (P-value 0.04).

Table 3 illustrates the results of intra-rater test, retest reliability of observation checklist for oncology nurses performance related to handling of chemotherapeutic agents. The data in this table shows that there is conformity between the results obtained in the first and second times of observation the only exception for the item " disposal of needles and syringes in special container" (P-value 0.003).

Table 4 demonstrates the results of intra-rater test, retest reliability of observation checklist for oncology nurses performance related to assessment of infection, fever, anemia, and thrombocytopenia. The data in this table shows that there is agreement between the results obtained in the first and second times of observation.

Table 5 clarifies the results of intra-rater test, retest reliability of observation checklist for oncology nurses performance related to gastrointestinal problems. The data in this table shows that there is agreement between the results obtained in the first and second times of observation except for the following items: assessing fever /24hrs. (P-value 0.04 ), providing mouth care every 4 to 6 hours with normal saline (P-value 0.01 ) and avoiding lemon or glycerin swabs (P-value 0.03).

Table 6 indicates the results of intra-rater test, retest reliability of observation checklist for oncology nurses performance related to pulmonary and urinary systems. The data in this table shows that there is agreement between the results obtained in the first and second times of observation.

Table 7 reveals the results of intra-rater test, retest reliability of observation checklist for oncology nurses performance related to psychological problems and nutrition. The data in this table shows there is congruence between the results obtained in the first and second times of observation except for one item: "keeping the patient's room free from bad odor" (P-value 0.006)

Table 8 illustrates the results of intra-rater test, retest reliability of observation checklist for oncology nurses performance related to skin alteration and 
neurotoxicity. The data in this table shows that there is concord between the results obtained in the first and second times of observation except for the following items: persuading patients that these changes are reversible (P-value 0.03), advising the patients to use hat or turban to cover head during the period of hair loss (P-value 0.02), informing patients that changes in nail color is expected during chemotherapy (P-value 0.02).

Table 9 shows the results of intra-rater test, retest reliability of observation checklist for oncology nurses performance related to extravasation. The data in this table shows that there is concurrence between the results obtained in the first and second times of observation except for two items: trying to aspirate residual chemotherapy from the site and cannula ( $\mathrm{P}$ value 0.01 ) and observing pain regularly ( $\mathrm{P}$-value $<0.001)$.

Table 10 clarifies the results of intra-rater test, retest reliability of observation checklist for oncology nurses performance related to patients transfer to other departments. The data in this table reveals that there is a statistical significant difference between the results obtained in the first and second times of observation in two items: recording causes of patients transfer (P-value < 0.001), and instructing patients about side effects of chemotherapy (P-value 0.02).

\section{Discussion:}

The delivery of chemotherapy is primarily responsibility of oncology nurse; the nurse must have knowledge of pharmacology of antineoplastic agent, proper techniques of drugs preparation and administration systems, drug interactions and possible adverse effects. (Thompson et al, 2004; Padberg \& Padberg, 2005). In addition, before starting chemotherapy the nurse should assess the patient's knowledge of cancer and chemotherapy, determine the patient's general, physical health, prepare the patient and his/her family to manage anticipated side effects of chemotherapy and to report symptoms of potentially side effects early.

This study was constructed as descriptive study in an attempt to assess nurses perfomance for patients with cancer receiving chemotherapy at South Egypt Cancer Institute at Assiut University. The subjects of study were 65 staff nurses from male, female, and private oncology units at South Egypt Cancer Institute. Data was collected using an observation checklist of nurses' performance.

Concerning the reliability of the proposed tool, it was tested in the present study through intra observer test retest method. The findings showed high intra observer reliability as evidence by the absence of statistically significant difference between the findings obtained in the first and second times of observation. However, there were significant statistical differences for some items in relation to the nurses' performance these findings were confirmed by (Saad, 2004).

In relation to the assessment and investigation, the findings of the present study indicated that there was statistical significance difference between the results obtained in the first and second times of observation for the following items e.g. assess height, temperature, heart disease, obtain specimen accurately, and record time of obtaining specimen. Although all nurses in study setting don't assess plus. These findings are inconsistent with Hilkemeys (2005) who stressed that the nurse should perform complete physical and emotional assessment for the patients with cancer.

Concerning nurses' performance related to handling chemotherapeutic agents, the results showed consistency of the tool except for, dispose needles and syringe in special container, the results revealed that all nurses don't wear mask to cover nose and mouth and eye protector. this in contrast with Daniels (2004) who emphasized that used needles should not be recapped, bent, or broken; needles should be placed in a puncture resistant marked or color, coded container close to the work site, correct disposal decrease the risk of needle punctures to caregivers, replace container when two thirds full. Moreover, Central for Disease Control and Prevention (CDC) (2009) reported that standards precautions must be practiced with all patients since this is the most effective method to decrease the risk of infection for patients and care givers.

Although, the results showed consistency of the tool regarding to nursing care for patients with cancer have infection, fever, anemia, and thrombocytopenia, the results showed that the majority of the nurses in study setting don't perform the majority of nursing activities. This finding disagree with the findings of Linton \& Maehius (2003) who pointed out that the patients with cancer receiving chemotherapy become at risk of infection, and monitoring blood cell count frequently is essential, as it is protecting the patient from infection and injury.

The study findings found a statistical significant difference between the results obtained in the first and second times of observation in relation to nursing care for patients with cancer receiving chemotherapy as regards to gastrointestinal tract complication in regard to observing signs of fever/24hrs, providing mouth care every 4 to 6 hours, and avoiding lemon or glycerin swabs. The present results showed that the patients with cancer receiving chemotherapy have gastrointestinal complication don't receive complete nursing care because the nurses at assigned setting 
were not knowledgeable about the management of gastrointestinal problems, inspite of this nursing care considered a critical care procedures for patients with cancer receiving chemotherapy. This finding is not consistent with Christman (2008) who mentioned that most patients with cancer receiving chemotherapy treated with radiation or chemotherapy prone to nausea and vomiting, the nurse should assess characteristics of vomiting through observing its amount, color, blood, and foreign substances. In addition Strohi (2004) stressed that the nurse should inspect the patient's mouth daily, provide mouth care every 4 to 6 hours with normal saline, avoid lemon or glycerin swabs, apply a topical viscous anesthetic as order, recommended patient to take a soft bland diet and report any change to the physician.

The results showed consistency of the study tool regarding nursing care for patients with cancer receiving chemotherapy have pulmonary and urinary complication in the results obtained in the first and second times of observation. However, it was clear that large number of nurses ignored the nursing activities related to pulmonary and urinary systems. This might be attributed to nurses' shortage, this is in contrast with Ostahega (2002) who stated that the nurse must auscultate lungs of the patient, observe shortness of breath, cough and presence of sputum, facilitate respiratory effort and encourage deep breathing. In addition, Bernier (2003) reported that the observation and measuring intake and output are very important to prevent complication and is mainly nurse responsibility.

In the same way the results showed consistency of the tool regarding the psychological nursing care for patients with cancer receiving chemotherapy. It was revealed that the nurses' performance was inadequate. This is in contrast with Brunner \& Lwomoto (2005) stated that the nurse has an important role to satisfy the psychological needs of both patients and his/her family as it let them to talk freely about their problems

As regards to the nursing care for improving nutrition for patients with cancer receiving chemotherapy, the results showed consistency of the tool in the first and second times of observation, except for the item related to keeping patient room free from bad odor. The present findings revealed that the majority of nurses don't perform nursing activities related to improving nutrition for the patient receiving chemotherapy; this may be due to lack of policies and procedures and all nurses in the present study beliefs that nutritional instruction is the responsibility of the medical staff. This is in contrast with Cotanch (2008)) who emphasized that the patients with cancer need nutrients necessary for normal tissue healing and resistance to infection. The patient who is malnourished is at higher risk for alteration in the fluids and electrolyte and infection.

It was found from the present study findings that there is a statistical significant difference between the results obtained in the first and second times of observation in relation to nursing care for patients with cancer receiving chemotherapy having skin alteration and neurotoxcity for the following items: persuading patient that these changes are reversible, advising patient to use hat or turban to cover head during period of hair loss, and informing patients that change in nail color is expected during chemotherapy. The results showed that the nursing care provided for patients with cancer have skin alteration and neurotoxcity were inadequate. This is in contrast with Tennebaum (2006) who stated that the nurse must inspect the patient's skin, and bony prominence for any change, such as depended edema, change in color of the skin such as, pallor, redness, hotness, coldness or breakdown to find out any abnormalities. Additionally, Keline (2005) asserted that the oncology nurse must observe, monitor and report weakness, numbness, and tingling sensation of patient hand, arms and feet.

Regarding the nursing care for patient with cancer when extravasation occurs the results showed a consistency of the study tool, however there is a statistical significant difference for two items namely; try to aspirate residual of chemotherapeutic agent from the site and cannula, and observe pain. In contrast with these findings, Ruppens (2007) recommended that the nurse must provide immediate and specific nursing care for patients with cancer when extravasation occur.

With regard to nursing care for patients with cancer receiving chemotherapy when discharged from hospital, the results showed a consistency of the study tool, Meanwhile, the majority of nurses don't provide instructions for patients with cancer at discharge from the hospital and they don't provide instructions for patient about sexual and reproductive function, even if they have a time to give such instructions, This finding in contrast with Rausahi and Turhoski (2003). Who stated that patient education is a major professional responsibilities, major component of most nursing care standards and mandated part of nursing activities in most acute care institutions.

\section{Conclusion:}

Based on the results of the study, it can be concluded that:

- The nursing care provided for patients with cancer receiving chemotherapy were inadequate in some aspects of nursing care such as, psychological care, discharge instructions and so on. 
- In order to provide good quality nursing care for patients with cancer receiving chemotherapy, this requires that the oncology unit should be staffed with qualified, adequate nurses who are to perform assessment and able to provide adequate nursing intervention during three day shifts according to patients needs and standard of care.

\section{Recommendations:}

Based on the results of this study, emphasis should be directed to the following recommendations.

1-Periodical appraisal of nurse's performance should be done with continuous feedback, and correction for their performance deviation.

2-Informing the nurses about the importance of assuring emotional and psychological status of patients as well as physical and health status.

3-Nurses working should be involved in regular inservice training programs to improve their skills and update their knowledge according to the latest advances.

4-Further studies must be done to measure quality of care and also to develop nursing care standards for patient with cancer.

5-Continuing the supervision of nurses for detecting the performance weakness to be identified and corrected.

\section{References}

1. American Cancer college of Surgeons. Commission on Cancer Program Standards. (2004): A Available at: Http://www. Face org/cancer/program standards.html.

2. Association Commission Cancer Center Guidelines. (2006): Clinical Management and Supportive Care Services, Oncology Nursing Society Position, P. 709.

3. Day G.K., and Adjei A.A. (2006): Principles of Chemotherapy; In Chang A.E., Ganz P.A., Hayes D.F., Kinsella T.J., Paus H.I., Schiller J.H., Stone R.M., and Strecher V.J (eds). Oncology: An evidence based approach. 1st ed Springer science+ business Media. Inc New York, USA, Pp. 14-40.

4. Hilkemeys. J. (2005): The Preoperative Assessment and Postoperative Surveillance of Patient with Cancer, Surgical Clinic of North America, 8 (5): Pp. 1070-1072.

5. Linton, A.B., and Maehius N. (2003): Introduction to Medical Surgical Nursing; 3rd ed, Saunders Company, Philadelphia, Pp. 330346.

6. Saad, N.F. (2004): Developing Nursing Care Standard for Stroke Patient, thesis Submitted for Partial Fulfillment of the Requirement of Doctor Degree in Nursing Science, Faculty of Nursing.
Ain Shams University, unpublished, p. 116-117.

7. Strohi R.A. (2004): Radiation Therapy, Recent Advances and Nursing Implications. Nurse Clin. N Amer. 35 (12): P. 409.

8. -Bernier E. (2003): Nutritional Problems Associated with Gastrointestinal and Genitourinary Cancer. Cancer Research, 57 (45): P.260

9. -Brunner L. S., and Lwomoto R. (2005): Altered Sexual Health. In Cancer Nursing, New York: W M C Brow Publishers, P. 642.

10. -Cancer Care Ontario (Formerly Ontario Cancer treatment and research Foundation. (1996): Standards for Provincial Systemic Treatment Program. Toronto. P. 7.

11. -Center for Disease Control and Prevention. (2009): Available at http:www.ed.c.gov Amcancers.org/cgi/content/ful/55/1/10).

12. -Christman N. (2008): Uncertainly and Adjustment during Radiotherapy, Nursing Research, 118 (35): Pp. 19-20.

13. -Cotanch P. (2008): Relaxation Training for Control of Nausea and Vomiting in Patient Receiving Chemotherapy, Cancer Nurse Research, Pp. 277-283.

14. -Daniels E. (2004): Nursing Fundamentals and Clinical Decision Making Patient with Cancer, Surgical Clinic of North America, 8 (5): Pp. 1070-1072.

15. -Hargrove S., and Derstine. J.B. (2006): General Concepts in Rehabilitation Nursing. W.B. Saunders Company, P. 66-68.

16. -Joint Commission on Accreditation of Health Care Organization (2005): Monitoring and Evaluation of the Quality and Appropriateness of Care: A Hospital Example. Quality Review Bulletin; Pp. 236-330.

17. -Keline $\boldsymbol{E}$. (2005): Concern of Terminally ill Adults Cancer Patients, Cancer Nursing, Pennsylvania: Sprinhouse co., p164-171.-

18. -Mohamed B. (1999): Impact of Designed Self Instructional Module on Knowledge and Compliance of Patients Receiving Chemotherapy, Submitted in Partial Fulfillment of Doctorate Degree in Medical Surgical Nursing Assiut University, unpublished, p. 140.

19. -Ostchega Y. (2002): Prevention and Treatment Cancer Chemotherapy Complications, Nursing Research, 15 (52), P. 47-53.

20. -Padberg R.M., and Padberg L.F. (2005): Patient Education and Support in Cancer Nursing Principles and Practice 7thed. New Jersey: Appleton and Long, Pp. 1609-1613.

21. -Rousahi, M., and Turhoski; B (2003): Developing Realistic Treatment Standards in Today's Economic Climate: Stroke Survivor 
Education. Journal of Advanced Nursing; 30 (5): Pp.329-334.

22. -Rupens R.D. (2007): Chemotherapy and Management of Special Problems, Nurse Time 31, p1.336.

23. -Sabra H. (2003): Assessment of Nurses Knowledge and Nursing Intervention for Cystectomy Patient, unpublished master thesis Nursing science, Faculty of Nursing. Assuit University, P. 61.

24. -Tennebaum L. (2006): Cancer Chemotherapy and Biotherapy 4th ed, Philadelphia PA: WB Saunders, P. 402.

25. -Thompson M.J, Babiera, G.V., Skoracki, R.J, \& Esteva, F.J. et al. (2004): The Influence of Finasteride on the Development of Prostate Cancer. N Engl J Med, 349 (3): Pp215-24.

26. -Vogel V., and Costantino J. (2006): Effects of Tamoxifen v5 Rafxifene on the Risk of Developing (STAR) p-2 Trail. JAMA 295 (23), Pp. 2727-41. PMID 167527 (http:www.ncbi-nlm.nih.gov/entrez/query- fcgi).

27. -Wikipedia E. (2009): Cancer Classification and External Resources. Retrived from http:// em.Wikipedia. org/ wiki/ cancer. 